\title{
Seletividade de produtos naturais comerciais a Trichogramma pretiosum (Riley, 1879) (Hymenoptera: Trichogrammatidae)
}

\author{
Daiane Luckmann ${ }^{2}$, Alfredo de Gouvea ${ }^{3}$, Michele Potrich ${ }^{4}$, Everton Ricardi Lozano da Silva ${ }^{5}$, \\ Barbara Puretz ${ }^{6}$, Sidinei Dallacort ${ }^{7}$, Thiago Evandro Gonçalves ${ }^{8}$ \\ http://dx.doi.org/10.1590/0034-737X201461060006
}

\begin{abstract}
RESUMO
A seletividade de produtos naturais a parasitoides é pouco conhecida. Em vista disso, este trabalho teve por objetivo avaliar a seletividade de produtos naturais comerciais a Trichogramma pretiosum Riley (Hymenoptera: Trichogrammatidae). Os produtos utilizados foram Baicao ${ }^{\circledR}$, Orobor $^{\circledast}$ e Topneem ${ }^{\circledR}$, nas concentrações recomendadas pelos fabricantes, e pulverizados sobre cartelas contendo ovos esterilizados de Anagasta kuehniella Zeller (Lepidoptera: Pyralidae). Teste com chance de escolha para parasitismo foi realizado, confinando-se uma fêmea de T. pretiosum com duas cartelas, uma pulverizada com o produto e outra considerada testemunha, avaliando-se a percentagem de parasitismo. O teste sem chance de escolha consistiu na pulverização dos tratamentos, em cartelas, previamente ou posteriormente ao parasitismo. Em ambos os testes, avaliaram-se o número de ovos parasitados, a percentagem de emergência e a longevidade de adultos. No teste com chance de escolha, os produtos Topneem ${ }^{\circledR} \mathrm{e}$ Baicao ${ }^{\circledast}$ provocaram redução do parasitismo de T. pretiosum. No teste sem chance de escolha, Baicao ${ }^{\circledast}$, pulverizado previamente sobre cartelas contendo ovos de A. kuehniella, interferiu negativamente na emergência de T. pretiosum. Houve redução do número de ovos parasitados quando Baicao $^{\circledast}$ foi pulverizado no tratamento pré-parasitismo. Orobor $^{\circledast}$ é seletivo a $T$. pretiosum nas condições de realização do experimento e não afetou negativamente os parâmetros avaliados. Baicao ${ }^{\circledR}$ não foi seletivo para T. pretiosum, afetou a maioria dos parâmetros avaliados e foi classificado como levemente nocivo quanto à toxicidade aos adultos do parasitoide, em condições de laboratório.
\end{abstract}

Palavras-chave: compatibilidade, parasitoide, organismo não alvo.

\section{ABSTRACT}

\section{Selectivity of commercial natural products to Trichogramma pretiosum (Riley, 1879) (Hymenoptera: Trichogrammatidae)}

The selectivity of natural products to parasitoids is not well known. Thus, the objective of this work was to evaluate the selectivity of this commercial natural products to Trichogramma pretiosum Riley (Hymenoptera: Trichogrammatidae). The products used in the experiment were the following: Baicao ${ }^{\circledR}$, Orobor $^{\circledR}$ and Topneem $^{\circledast}$ at doses recommended by the manufacturers and were sprayed on cards containing sterilized eggs of Anagasta

\footnotetext{
Recebido para publicação em 08/04/2013 e aprovado em 06/06/2014

${ }^{1}$ Este trabalho é parte da dissertação de mestrado da primeira autora.

${ }^{2}$ Tecnóloga em Horticultura. Programa de pós-graduação em Agronomia, Universidade Tecnológica Federal do Paraná, Campus Pato Branco, Via do Conhecimento, km 01, Pato Branco, Paraná, Brasil. daianeluck@ yahoo.com.br (autora para correspondência)

${ }^{3}$ Licenciado em Ciências Agrárias, Doutor. Diretor de Graduação, Universidade Tecnológica Federal do Paraná, Campus Dois Vizinhos, Estrada para Boa Esperança, Km 04, 85660000, Dois Vizinhos, Paraná, Brasil. alfredo@utfpr.com.br

${ }^{4}$ Bióloga, Doutora. Coordenação de Ciências Biológicas, Universidade Tecnológica Federal do Paraná, Campus Dois Vizinhos, Estrada para Boa Esperança, Km 04, 85660-000, Dois Vizinhos, Paraná, Brasil. michelepotrich@utfpr.edu.br

${ }^{5}$ Biólogo, Doutor. Coordenação de Ciências Biológicas, Universidade Tecnológica Federal do Paraná, Campus Dois Vizinhos, Estrada para Boa Esperança, Km 04, Dois Vizinhos, 85660-000, Paraná, Brasil. evertonlricardi@utfpr.edu.br

${ }^{6}$ Graduanda no curso de Engenharia Florestal. Universidade Tecnológica Federal do Paraná, Campus Dois Vizinhos, Estrada para Boa Esperança, Km 04, 85660-000, Dois Vizinhos, Paraná,bah_puretz@hotmail.com

${ }^{7}$ Graduanda no curso de Engenharia Florestal. Universidade Tecnológica Federal do Paraná, Campus Dois Vizinhos, Estrada para Boa Esperança, Km 04, 85660-000, Dois Vizinhos, Paraná, Brasil. sidineidallacort@ hotmail.com

${ }^{8}$ Graduando do curso de Engenharia Florestal. Universidade Tecnológica Federal do Paraná, Campus Dois Vizinhos, Estrada para Boa Esperança, Km 04, 85660-000, Dois Vizinhos, Paraná, Brasil. thiagoevandrogoncalves@gmail.com
} 
kuehniella Zeller (Lepidoptera: Pyralidae). For the free-choice test, a T. pretiosumfemale was confined with two cards, one of them was sprayed with the natural products and the other was considered control, evaluating the percentage of parasitism. The no-choice test consisted in spraying treatments on cards previously or subsequently to parasitism. The number of parasitized eggs, percentage of emergence and longevity of adults were evaluated in both tests. Topneem ${ }^{\circledast}$ and $\mathrm{Baicao}^{\circledast}$ showed increase in parasitism of T. pretiosum in the free-choice test. Baicao ${ }^{\circledast}$ sprayed previously on cards containing eggs of A. kuehniellahad a negative effect on the emergency of T. pretiosum in the no-choice test. A reduction in the number of parasitizedeggs was found when Baicao ${ }^{\circledR}$ was sprayed previously in the parasitism treatment. Orobor ${ }^{\circledR}$ was selective to T. pretiosum in the experiment conditions and it did not affect negatively the parameters evaluated. Baicao ${ }^{\circledR}$ was not selective to T. pretiosum, it affected negatively most of the parameters evaluated, and classified as slightly harmful for toxicity to $T$. pretiosum adults under laboratory conditions.

Key words: compatibility, non-target organism, parasitoid.

\section{INTRODUÇÃO}

A manutenção de parasitoides nos agroecossistemas é importante como fator de equilíbrio dinâmico das populações de espécies de insetos. Observa-se, com frequência, o controle biológico natural, exercido por inimigos naturais com potencial de manterem populações de pragas em níveis aceitáveis, o que permite diminuir a intervenção para o seu controle (Degrande et al., 2002).

O parasitoide de ovos Trichogramma (Hymenoptera: Trichogrammatidae) é um dos agentes de controle biológico mais difundido, utilizado e comercializado no Brasil, parasitando as ordens Lepidoptera, Hemiptera e Coleoptera (Gonçalves et al., 2003; Parra \& Zuchi, 2004). Este gênero de parasitoides destaca-se como um dos principais no controle de insetos da ordem Lepidoptera, como Tuta absoluta (Faria et al.,2008), Spodoptera frugiperda (Smith), Spodoptera albula (Walker), Spodoptera eridania (Cramer), Anticarsia gemmatalis Hubner, Pseudoplusia includens (Walker), Diatraea saccharalis (Fabricius) (Siqueira et al., 2012), entre outras.

A utilização de agentes de controle biológico ocorre conjuntamente ou na sequência ao uso de produtos fitossanitários e, no caso dos sistemas alternativos de produção, ao dos produtos naturais, como extratos vegetais, óleos essenciais (Gallo et al., 2002; Penteado, 2007) ou caldas à base de cobre e enxofre. Embora esses produtos sejam menos tóxicos ao homem e ao ambiente que os convencionais produtos químicos utilizados, podem apresentar repelência, efeito comportamental e/ou, biológico em inimigos naturais.

Trabalhos têm sido desenvolvidos para avaliar o efeito de produtos conhecidos como alternativos, botânicos ou naturais, sobre Trichograma pretiosum Riley (Hymenoptera: Trichogrammatidae), com extratos aquosos e outros produtos à base de neem (Azadirachta in- dica A. Juss) (Oliveira et al., 2003; Gonçalves-Gervásio \& Vendramim, 2004, Hohmann et al., 2010; Silva, 2010), extrato pirolenhoso (Morandi Filho et al., 2006, Silva, 2010), extratos de asteráceas (Tavares et al., 2009), óleo de mamona (Bestete et al., 2011), extratos de mirtáceas (Purwatiningsih et al., 2012) e produtos botânicos comerciais (Silva, 2010).

Entretanto, apesar dos estudos existentes, informações sobre o efeito de produtos naturais comerciais sobre agentes de controle biológico são escassas, pelo constante advento de novos produtos no mercado. Neste sentido, os produtos Orobor $^{\circledR}$, Baicao ${ }^{\circledR}$ e Topneem ${ }^{\circledR}$, atualmente utilizados pelos produtores orgânicos e agroecológicos, apresentam a necessidade de maiores informações sobre seu espectro de ação, em especial sobre organismos não alvos. Diante do exposto, o objetivo deste trabalho foi avaliar a seletividade destes produtos naturais comerciais sobre $T$. pretiosum.

\section{MATERIAL E MÉTODOS}

O trabalho foi desenvolvido no Laboratório de Controle Biológico da Universidade Tecnológica Federal do Paraná, Campus Dois Vizinhos (UTFPR-DV).

Para isto, ovos de Anagasta kuehniella (Lepidoptera: Pyralidae), esterilizados e parasitados por T. pretiosum, foram fornecidos pelo Laboratório de Controle Biológico, do Centro de Ciências Agrárias, da Universidade Estadual do Oeste do Paraná - Unioeste, Campus Marechal Cândido Rondon, PR, Brasil.

Os produtos naturais comerciais Orobor $^{\circledR}$, Baicao ${ }^{\circledR}$ e Topneem ${ }^{\circledR}$ (Tabela 1), certificados para diferentes culturas de sistemas orgânicos de produção, foram obtidos em loja especializada na venda de produtos naturais e/ ou, alternativos. As caldas dos produtos naturais, na concentração recomendada pelo fabricante (Tabela 1), foram preparadas em frascos erlenmeyers, contendo 100 $\mathrm{mL}$ de água destilada esterilizada. 
Cartelas $(5,0 \times 1,0 \mathrm{~cm})$ com aproximadamente 200 ovos de A. kuehniella, esterilizados e não parasitados, foram utilizadas para os testes realizados. Foram arranjadas aos pares sobre a bancada e pulverizadas com cada tratamento, com aerógrafo Pneumatic Sagyma ${ }^{\circledR}$, acoplado a uma bomba de pressão Fanem ${ }^{\circledR}$, regulada a 1,2 kgf / $\mathrm{cm}^{2}$; o volume de calda utilizado foi de $200 \mu \mathrm{L}$, suficiente para cobrir as cartelas.

\section{Teste com chance de escolha: pulverização pré-parasitismo}

Cada tratamento constou da pulverização de água destilada esterilizada (testemunha), sobre 20 cartelas, e da pulverização da calda de um produto natural, sobre outras 20 cartelas. Após a pulverização, uma cartela pulverizada com água destilada esterilizada e uma cartela pulverizada com a calda do produto natural, devidamente identificadas, foram dispostas no interior de um tubo de vidro de fundo chato $(10 \mathrm{~cm} \times 2,5 \mathrm{~cm})$, previamente esterilizado, totalizando 20 (tubos) repetições por tratamento, conforme metodologia adaptada de GonçalvesGervásio \& Vendramim (2004). Juntamente com as cartelas foi acondicionada uma fêmea de T. pretiosum (com 24 h de emergência). Os tubos de vidro foram vedados com filme de PVC, acondicionados, por $24 \mathrm{~h}$, em câmara climatizada tipo BOD (temperatura de $26 \pm 2{ }^{\circ} \mathrm{C}$, UR: $70 \pm 10 \%$ e $14 \mathrm{~h}$ de fotofase) e as fêmeas retiradas após esse período. $\mathrm{O}$ mesmo procedimento foi realizado para todos os produtos.

A percentagem de parasitismo por T. pretiosum foi avaliada, após 96 horas, comparando-se cada produto natural com sua respectiva testemunha, o somatório do número de ovos parasitados em ambos (testemunha e tratamento) considerado como $100 \%$, seguindo-se a equação POPTrat $=\frac{\text { OPTrat }}{\text { OPTrat }+ \text { OPTest }} \times 100$, em que POPTrat $=$ percentagem de ovos parasitados no tratamento, OPTrat = ovos parasitados no tratamento e OPTest $=$ ovos parasitados na testemunha (Potrich, 2010).

Os dados de percentagem de parasitismo foram submetidos à análise de duas amostras relacionadas por meio do teste não paramétrico Wilcoxon, com o programa estatístico Bioestat 5.0 (Ayres et al., 2007).

\section{Teste sem chance de escolha: Pulverização pré-parasitismo}

Cartelas com ovos de A. kuehniella, esterilizados e não parasitados, foram pulverizados com as caldas dos produtos naturais, sendo utilizadas 20 cartelas (repetições) para cada tratamento. Em seguida, as cartelas foram individualizadas, juntamente com uma fêmea de $T$. pretiosum, com $24 \mathrm{~h}$ de emergência, em tubos de vidro de fundo chato $(10 \mathrm{~cm} \times 2,5 \mathrm{~cm})$, previamente esterilizados. O acondicionamento ocorreu nas mesmas condições descritas no teste com chance de escolha. Após 24 $\mathrm{h}$, as fêmeas foram retiradas dos tubos e depois de $96 \mathrm{~h}$ foi realizada a avaliação. A testemunha constou da pulverização de água destilada esterilizada.

O número de ovos de A. kuehniella parasitados por T. pretiosum foi avaliado, contando-se os ovos enegrecidos, que correspondem à fase de pré-pupa desse parasitoide, quando apresenta grânulos de urato, concentrados no abdome (Cônsoli et al., 1999). O percentual de emergência e a longevidade de machos e fêmeas também foram parâmetros avaliados. A redução da capacidade de parasitismo para fêmeas dos tratamentos foi comparada com a da testemunha e calculada pela equação $R P=\left[1-\left(\frac{\mathrm{P}}{\mathrm{p}}\right) * 100\right]$, em que RP = Percentagem de redução do parasitismo, $\mathrm{P}=$ valor do parasitismo médio em cada tratamento, $\mathrm{p}=$ parasitismo médio observado na testemunha.

Com base na capacidade de redução do parasitismo, os produtos naturais foram classificados em quatro categorias, conforme "International Organization for Biological Control" (IOBC): 1 = inócuo $(<30 \%), 2$ = levemente nocivo $(30-79 \%), 3=$ moderadamente nocivo $(80-99 \%), 4$ = nocivo (> 99\%) (Hassan, 1997). O percentual de emergência foi calculado pela equação $P e=\frac{T e}{T o} \times 100$, em que $P e=$ Percentagem de emergência, $T e=$ Total de emergidos, $T o=$ Total de ovos

Tabela 1. Produtos naturais comerciais, recomendação de uso, composição e concentração recomendada, conforme dados fornecidos pelo fabricante

\begin{tabular}{|c|c|c|c|}
\hline Produto & Uso & Composição & Conc. \\
\hline Orobor ${ }^{\circledR}$ & $\mathrm{FF}$ & $\begin{array}{c}\text { Óleo da casca de laranja (Citrus sinensis) enriquecido com elementos } \\
\text { essenciais Boro e Nitrogênio }\end{array}$ & $0,005 \mathrm{~L} / 1000 \mathrm{~L}$ \\
\hline Baicao $^{\circledR}$ & $\mathrm{FF}$ & Raiz de Timbó (Derris sp.) & $0,0015 \mathrm{~L} / 1000 \mathrm{~L}$ \\
\hline Topneem $^{\circledast}$ & $\begin{array}{l}\text { INS, FUN e } \\
\quad \text { BAC }\end{array}$ & $\begin{array}{c}\text { Óleo de neem (folhas, torta de sementes, e óleo), } \\
\text { rotenona (timbó), piretro natural (crisântemo), extrato pirolenhoso } \\
\text { (eucalipto), alho (Allium sativum), alamanda (Allamanda blanchetti), } \\
\text { maravilha (Mirabilis jalapa), cinamomo (Melia azedarach) } \\
\text { e pimenta do reino (Piper nigrum) }\end{array}$ & $0,01 \mathrm{~L} / 1000 \mathrm{~L}$ \\
\hline
\end{tabular}


parasitados (Potrich, 2010). A média da longevidade foi calculada por meio de média ponderada.

Os dados foram submetidos à análise de variância, as médias comparadas entre si, pelo teste não paramétrico de Kruskal-Wallis, e a relação entre os tratamentos (pré e pós-parasitismo) comparados pelo teste não paramétrico de Mann-Whitney (duas amostras independentes), como programa estatístico Bioestat 5.0 (Ayres et al., 2007).

\section{Teste sem chance de escolha: Pulverização pós-parasitismo}

Uma fêmea de T. pretiosum com, no máximo 24 h de emergência, foi individualizada em tubo de vidro de fundo chato $(10 \mathrm{~cm} \times 2,5 \mathrm{~cm})$, com cartela contendo ovos esterilizados de A. kuehniella. O acondicionamento ocorreu nas mesmas condições descritas nos testes anteriores. A fêmea foi retirada após $24 \mathrm{~h}$ e a cartela pulverizada com a calda do produto natural, sendo utilizadas 20 cartelas (repetições) por tratamento. A testemunha foi pulverizada com água destilada esterilizada. Os tubos retornaram para a câmara climatizada, nas mesmas condições descritas. Os parâmetros avaliados, bem como a análise dos dados, foram os mesmos descritos no teste de pré-parasitismo.

\section{RESULTADOS E DISCUSSÃO}

\section{Teste com chance de escolha: pulverização pré-parasitismo}

Orobor $^{\circledR}$ não provocou redução do parasitismo de $T$. pretiosum, não diferindo de sua respectiva testemunha. Baicao $^{\circledR}\left(35,8 \%\right.$ de parasitismo) e Topneem ${ }^{\circledR}(24,4 \%$ de parasitismo) provocaram redução do parasitismo de $T$. pretiosum, diferindo das respectivas testemunhas $(64,2$ e 75,6\%) (Tabela 2). Esse efeito pode ter sido provocado pelo fato de as fêmeas de $T$. pretiosum terem a capacidade de identificar substâncias tóxicas ou repelentes sobre ovos do hospedeiro, podendo não parasitá-los (Vinson, 1997).

Baicao $^{\circledR}$ apresenta rotenona em sua composição e essa substância pode ter sido responsável pela redução do parasitismo. Esse princípio ativo, avaliado em teste de livre escolha para preferência alimentar de adultos de Apis mellifera (Hymenoptera: Apidae), mostrou efeito tóxico e repelente (Xavier, 2009). Entretanto, Rotenat ${ }^{\circledR}$ (produto formulado comercialmente e que apresenta rotenona em sua composição), pulverizado sobre ovos de Euchistus heros (Fabricius) (Hemiptera: Pentatomidae) não repeliu o parasitismo de Telenomus podisi (Ashmead) (Hymenoptera: Scelionidae) (et al., 2013). A redução do parasitismo, causada por rotenona, pode estar relacionada com a ação desse composto como inibidor enzimático, interrompendo o metabolismo energético mitocondrial, o que reduz as taxas respiratórias e leva o animal à morte (Kathrina \& Antonio, 2004).

Topneem ${ }^{\circledR}$, cujo composto ativo predominante é a azadiractina, provocou redução do parasitismo de $T$. pretiosum. Esse não é um efeito positivo, no enfoque do controle biológico; no entanto, é um efeito comumente observado sobre insetos-praga, como observado sobre Plutella xylostella (L.) (Lepidoptera: Yponomeutidae) (Dequech et al., 2009) e Aphis gossypii Glover (Hemiptera: Aphididae) (Andrade et al. 2013).

Resultados análogos aos observados neste trabalho foram obtidos por Gonçalves-Gervásio \& Vendramim (2004), que verificaram que o extrato aquoso de sementes de Azadirachta indica A. Juss., na concentração de $10 \%$, repeliu $T$. pretiosum, reduzindo o parasitismo em $85 \%$; entretanto, o produto formulado Pironin, avaliado por Silva (2010), não repeliu T. pretiosum não afetando, consequentemente, o parasitismo quando aplicado sobre ovos de A. kuehniella.

Azadiractina (Azamax 2,0 mL/L), sobre ovos de espécies da ordem Lepidoptera, resulta na gênese de tecidos alterados, ausência de desenvolvimento e de formação embrionar, grânulos de vitelo dispersos e desintegração do córion (Correia et al., 2013). Esses são fatores relevantes no processo de oviposição realizado pela fêmea de $T$. pretiosum, o que é, provavelmente, um fator preponderante na escolha dos ovos do hospedeiro.

\section{Teste sem chance de escolha: pulverização pré e pós - parasitismo}

Orobor $^{\circledR}$, Baicao ${ }^{\circledR}$ e Topneem $^{\circledR}$ não interferiram no desenvolvimento (número de ovos com sinal de parasitismo) de $T$. pretiosum, em ovos de A. kuehniella pulverizados após o parasitismo. Orobor ${ }^{\circledR}$ e Topneem ${ }^{\circledR}$ também não interferiram quando pulverizados previamente ao

Tabela 2. Percentagem ( \pm EP) de ovos de A. kuehniella parasitados por T. pretiosum, teste com chance de escolha entre ovos pulverizados com produtos naturais e água destilada esterilizada (temp. $26 \pm 2 \mathrm{C}$ e $14 \mathrm{~h}$ de fotofase)

\begin{tabular}{lc}
\hline Tratamento & Ovos parasitados $(\%)$ \\
\hline Testemunha $^{(\%)}$ & $64,2 \pm 8,44 \mathrm{a}$ \\
Baicao $^{\circledR}$ & $35,8 \pm 8,44 \mathrm{~b}$ \\
\hline$P$ & 0,0546 \\
\hline Testemunha & $75,6 \pm 7,81 \mathrm{a}$ \\
Topneem & $24,4 \pm 7,81 \mathrm{~b}$ \\
\hline$P$ & 0,002 \\
\hline Testemunha $^{\text {Orobor }}$ & $44,6 \pm 10,68 \mathrm{a}$ \\
\hline$P$ & $55,4 \pm 10,68 \mathrm{a}$ \\
\hline
\end{tabular}

Médias seguidas pela mesma letra, minúscula na coluna, não diferem entre si pelo teste Wilcoxon $(\mathrm{p}<0,05)$. 
parasitismo. No entanto, Baicao ${ }^{\circledR}$, pulverizado sobre ovos de A. kuehniella previamente, reduziu o número de ovos parasitados por T. pretiosum (7,4 ovos parasitados), quando comparado à testemunha, (26,7 ovos parasitados) (Tabela 3). Essa redução do parasitismo pode estar relacionada com a capacidade de identificar substâncias tóxicas, conforme destacado anteriormente (Vinson, 1997). Outra hipótese é de que o produto pode ter ocasionado a morte das fêmeas que estavam confinadas ao parasitismo, impedindo assim o maior número de ovos parasitados, o que pôde ser observado em alguns tratamentos, não sendo, porém, avaliado neste trabalho.

Os produtos à base de neem normalmente são descritos como repelentes ao parasitoide Trichogramma, ao contrário do que foi observado neste teste, quando o parasitoide encontra-se confinado. Neste caso, mesmo com a presença do produto e possível desintegração do córion, $T$. pretiosum realizou o parasitismo a fim de manter a progênie. O óleo emulsionável de neem, nas concentrações 0,33 , 0,53 e $1 \%$, provoca repelência total do parasitismo de Trichogramma galloi Zucchi sobre ovos de Diatraea saccharalis (Fabr.) (Lepidoptera: Crambidae), em teste sem chance de escolha (Broglio-Micheletti et al., 2006). O inseticida comercial AzaMax ${ }^{\circledR}$, nas concentrações de 0,2 e $0,0008 \%$, afeta a percentagem de parasitismo de $T$. pretiosum sobre ovos de Spodoptera frugiperda (J. E. Smith) (Lepidoptera: Noctuidae) (Correia, 2012). No entanto, o parasitismo de Trichogramma papilionis (Nagarkatti) sobre ovos de Corcyra cephalonica (Stainton) (Lepidoptera: Pyralidae), pulverizados com Neemix ${ }^{\circledR}$ não é reduzido (Sidi et al., 2012).

A variação observada nos resultados dos diferentes trabalhos com azadiractina pode estar relacionada com o tipo de produto ou sua formulação, pois o óleo pode ter provocado redução do número de ovos parasitados, por apresentar maior concentração desse princípio ativo (Menezes, 2005; Hohmann et al., 2010). O produto Topneem ${ }^{\circledR}$, testado neste trabalho, apresenta em sua composição os princípios ativos rotenona (Derris sp.), piretro natural (Chrysanthemum morifolium), extrato pirolenhoso de eucalipto (Eucalyptus sp.), alicina (Allium sativum), iridoides (Allamanda blanchetti), maravilha (Mirabilis jalapa), azaridina (Melia azedarach) e piperidina (Piper nigrum) (MFRural, 2011), apresentando menores concentrações de azadiractina pura, quando comparado com os demais produtos.

Baicao $^{\circledR}$, pulverizado diretamente sobre ovos de $A$. kuehniella, previamente à exposição ao parasitismo, reduziu o número de ovos parasitados por T. pretiosum. A aplicação do produto Orobor ${ }^{\circledR}$ após o parasitismo, quando comparada com a aplicação previamente ao parasitismo, reduziu o número de ovos com sinal de parasitismo.

Orobor $^{\circledR}$ e Topneem $^{\circledR}$ reduziram em menos de $30 \%$ o parasitismo das fêmeas de T. pretiosum e foram classificados como inócuos (classe1), segundo a IOBC (Hassan, 1997). Resultados similares são relatados para Natuneem (óleo de neem) e Biopirol (extrato pirolenhoso), considerados seletivos para $T$. pretiosum (Morandi Filho et al., 2006).

No entanto, Baicao ${ }^{\circledR}$ provocou redução da capacidade de parasitismo de fêmeas de T. pretiosum em 72,28\%, e foi classificado como levemente nocivo (classe 2) (Hassan, 1997). Em sistema agroecológico, em que se utiliza associação de produtos naturais e parasitoides para controle de diversos insetos praga, essa redução do parasitismo pode afetar consideravelmente o controle de pragas.

A emergência de adultos de T. pretiosum não foi afetada por Orobor ${ }^{\circledR}$ e Topneem ${ }^{\circledR}$, tanto na pulverização préparasitismo quanto na pós-parasitismo (Tabela 4). Resultados semelhantes foram encontrados para os produto comerciais à base de azadaractina, Dalneem, nas concentrações $0,25,0,5$ e 2,5\%, Pironim e Natural neem pulverizados antes da exposição dos ovos de $A$. kuehniella às fêmeas de T. pretiosum (Hohmann et al. 2010;. Silva, 2010) .

Tabela 3. Número médio ( \pm EP) de ovos de Anagasta kuehniella parasitados por Trichogramma pretiosum, pulverizados com produtos naturais prévia ou posteriormente ao parasitismo (temp. $26 \pm 2 \mathrm{C}$ e $14 \mathrm{~h}$ de fotofase)

\begin{tabular}{lccccc}
\hline \multicolumn{5}{c}{ Número de ovos parasitados por $\boldsymbol{T}$. pretiosum } \\
\hline Tratamentos & Pós-parasitismo* & Pré-parasitismo & $\boldsymbol{P}$ & RP $^{\mathbf{1}}$ & Classes $^{*}$ \\
\hline Testemunha $^{*}$ & $26,4 \pm 2,13 \mathrm{Aa}$ & $26,7 \pm 0,05 \mathrm{Aa}$ & 0,4057 & - & - \\
Orobor $^{\circledR}$ & $17,7 \pm 2,85 \mathrm{Ba}$ & $30,0 \pm 0,02 \mathrm{Aa}$ & 0,0049 & 0,00 & 1 \\
Baicao $^{\circledR}$ & $22,8 \pm 3,39 \mathrm{Aa}$ & $7,4 \pm 0,13 \mathrm{Bb}$ & 0,0003 & 72,28 & 2 \\
Topneem & $22,4 \pm 3,47 \mathrm{Aa}$ & $19,0 \pm 0,07 \mathrm{Aab}$ & 0,2347 & 28,84 & 1 \\
\hline$P$ & 0,3987 & 0,001 & &
\end{tabular}

${ }^{1} \mathrm{RP}=$ Redução na capacidade de parasitismo comparada com a testemunha; Classes da IOBC/WPRS para teste de toxicidade inicial sobre adultos: 1 = inócuo $(<30 \%), 2$ = levemente nocivo $(30-79 \%), 3=$ moderadamente nocivo $(80-99 \%), 4=$ nocivo $(>99 \%)$.

Médias seguidas pela mesma letra, minúscula na coluna, não diferem entre si pelo teste Kruskal-Wallis $(\mathrm{p}<0,05)$.

Médias seguidas pela mesma letra, maiúscula na linha, não diferem entre si pelo teste Mann-Whitney $(\mathrm{p}<0,05)$.

Rev. Ceres, Viçosa, v. 61, n.6, p. 924-931, nov/dez, 2014 
Observou-se redução da emergência de adultos de $T$. pretiosum, quando os ovos de A. kuehniella foram pulverizados com Baicao $^{\circledR}$, previamente e posteriormente à exposição ao parasitismo. Baicao ${ }^{\circledR}$ pulverizado previamente ao parasitismo afetou a emergência. Assim, sua pulverização previamente ao parasitismo é ainda mais agressiva ao desenvolvimento de $T$. pretiosum (Tabela 4).

Constatou-se aumento da longevidade média de fêmeas de $T$. pretiosum, emergidas de ovos de $A$. kuehniella, pulverizados previamente e posteriormente com Baicao ${ }^{\circledR}$. Aumento da longevidade dos machos foi observada apenas no tratamento de pré-parasitismo. Orobor $^{\circledR}$ e Topneem ${ }^{\circledR}$, pulverizados prévia ou posteriormente à exposição ao parasitismo, não interferiram na longevidade do parasitoide (Tabela 5).

Topneem pulverizado previamente ao parasitismo provocou redução da longevidade de machos de $T$. pretiosum. Efeito semelhante foi observado com produto comercial NIM-I-GO a $400 \mathrm{~mL} / 20 \mathrm{~L}$ de calda (Agrydine S.A), com redução de até 50\% da longevidade das fêmeas (Oliveira et al., 2003).
A longevidade e vigor dos adultos emergidos podem estar relacionadas com a disponibilidade de nutrientes para desenvolvimento do parasitoide no ovo do hospedeiro. Parasitoides que se desenvolveram em ovos menos nutritivos ou nos quais os produtos naturais interferiram nessa disponibilidade, podem emergir menos vigorosos e com menor longevidade (Potrich, 2010).

Os produtos naturais afetaram alguns dos parâmetros biológicos de T. pretiosum em laboratório. No entanto, no campo, esses efeitos podem ser ainda menores, já que no laboratório é forçado o contato. No campo, muitas vezes, os produtos podem não entrar em contato direto com o ovo do inseto-praga (Potrich et al., 2009).

Pode-se manejar o uso desses produtos, evitando-se aplicações simultâneas ou espaçando-se ao máximo possível suas pulverizações e a liberação do parasitoide. É importante salientar a necessidade de estudos em campo, avaliando-se, assim possíveis efeitos sobre adultos, além do efeito desses produtos na geração $\mathrm{F} 1$ do parasitoide.

Tabela 4. Emergência ( \pm EP) de adultos de Trichogramma pretiosum de ovos de Anagasta kuehniella pulverizados com produtos naturais, previamente ou posteriomente ao parasitismo (Temp. $26 \pm 2^{\circ} \mathrm{C}$ e $14 \mathrm{~h}$ de fotofase)

\begin{tabular}{|c|c|c|c|}
\hline \multicolumn{4}{|c|}{ Emergência de $T$. pretiosum (\%) } \\
\hline Tratamentos & Pré - parasitismo & Pós - parasitismo & $P$ \\
\hline Testemunha & $80,2 \pm 10,27 \mathrm{Aa}$ & $75,8 \pm 4,22 \mathrm{Aab}$ & 0,3479 \\
\hline Orobor $^{\circledR}$ & $77,8 \pm 5,30 \mathrm{Aa}$ & $90,8 \pm 12,21 \mathrm{Aa}$ & 0,1343 \\
\hline Baicao $^{\circledR}$ & $39,8 \pm 9,17 \mathrm{Bb}$ & $57,5 \pm 5,17 \mathrm{Ab}$ & 0,0433 \\
\hline Topneem & $90,9 \pm 4,10 \mathrm{Aa}$ & $81,5 \pm 5,30 \mathrm{Aa}$ & 0,0851 \\
\hline$P$ & 0,002 & 0,0059 & \\
\hline
\end{tabular}

Médias seguidas pela mesma letra, minúscula na coluna, não diferem entre si pelo teste Kruskal-Wallis (p<0,05).

Médias seguidas pela mesma letra, maiúscula na linha, não diferem entre si pelo teste Mann-Whitney $(p<0,05)$.

Tabela 5. Longevidade média ( \pm EP) de fêmeas e machos de Trichogramma pretiosum emergidos de ovos de Anagasta kuehniella pulverizados com produtos naturais, previamente e posteriormente ao parasitismo (temp. $26 \pm 2 \mathrm{C}$ e $14 \mathrm{~h}$ de fotofase)

\begin{tabular}{|c|c|c|c|}
\hline \multicolumn{4}{|c|}{ Longevidade de fêmeas de $T$. pretiosum (dias) } \\
\hline Tratamentos & Pré-parasitismo & Pós-parasitismo & $\boldsymbol{P}$ \\
\hline Testemunha & $1,4 \pm 0,10 \mathrm{Ab}$ & $1,5 \pm 0,11 \mathrm{Ab}$ & 0,4401 \\
\hline Orobor $^{\circledR}$ & $1,6 \pm 0,07 \mathrm{Ab}$ & $1,5 \pm 0,12 \mathrm{Aab}$ & 0,4264 \\
\hline Baicao $^{\circledR}$ & $2,1 \pm 0,26 \mathrm{Aa}$ & $1,9 \pm 0,11 \mathrm{Aa}$ & 0,2214 \\
\hline Topneem & $1,3 \pm 0,07 \mathrm{Ab}$ & $1,4 \pm 0,10 \mathrm{Ab}$ & 0,1647 \\
\hline$P$ & 0,0016 & 0,0155 & \\
\hline \multicolumn{4}{|c|}{ Longevidade de machos de $T$. pretiosum (dias) } \\
\hline Tratamentos & Pré-parasitismo & Pós-parasitismo & $P$ \\
\hline Testemunha & $1,5 \pm 0,08 \mathrm{Ab}$ & $2,0 \pm 0,16 \mathrm{Aa}$ & 0,0026 \\
\hline Orobor $^{\circledR}$ & $1,7 \pm 0,11 \mathrm{Aab}$ & $1,7 \pm 0,13 \mathrm{Aa}$ & 0,4563 \\
\hline Baicao $^{\circledast}$ & $2,4 \pm 0,50 \mathrm{Aa}$ & $1,8 \pm 0,10 \mathrm{Aa}$ & 0,1767 \\
\hline Topneem & $1,4 \pm 0,10 \mathrm{Bb}$ & $1,9 \pm 0,16 \mathrm{Aa}$ & 0,0164 \\
\hline$P$ & 0,008 & 0,6126 & \\
\hline
\end{tabular}

Médias seguidas pela mesma letra, minúscula na coluna, não diferem entre si pelo teste Kruskal-Wallis $(\mathrm{p}<0,05)$.

Médias seguidas pela mesma letra, maiúscula na linha, não diferem entre si pelo teste Mann-Whitney $(\mathrm{p}<0,05)$. 
Tabela 5. Longevidade média ( \pm EP) de fêmeas e machos de Trichogramma pretiosum emergidos de ovos de Anagasta kuehniella pulverizados com produtos naturais, previamente e posteriormente ao parasitismo (temp. $26 \pm 2 \mathrm{C}$ e $14 \mathrm{~h}$ de fotofase)

\begin{tabular}{|c|c|c|c|}
\hline \multicolumn{4}{|c|}{ Longevidade de fêmeas de $T$. pretiosum (dias) } \\
\hline Tratamentos & Pré-parasitismo & Pós-parasitismo & $P$ \\
\hline Testemunha & $1,4 \pm 0,10 \mathrm{Ab}$ & $1,5 \pm 0,11 \mathrm{Ab}$ & 0,4401 \\
\hline Orobor $^{\circledR}$ & $1,6 \pm 0,07 \mathrm{Ab}$ & $1,5 \pm 0,12 \mathrm{Aab}$ & 0,4264 \\
\hline Baicao $^{\circledR}$ & $2,1 \pm 0,26 \mathrm{Aa}$ & $1,9 \pm 0,11 \mathrm{Aa}$ & 0,2214 \\
\hline Topneem & $1,3 \pm 0,07 \mathrm{Ab}$ & $1,4 \pm 0,10 \mathrm{Ab}$ & 0,1647 \\
\hline$P$ & 0,0016 & 0,0155 & \\
\hline \multicolumn{4}{|c|}{ Longevidade de machos de $T$. pretiosum (dias) } \\
\hline Tratamentos & Pré-parasitismo & Pós-parasitismo & $P$ \\
\hline Testemunha & $1,5 \pm 0,08 \mathrm{Ab}$ & $2,0 \pm 0,16 \mathrm{Aa}$ & 0,0026 \\
\hline Orobor $^{\circledR}$ & $1,7 \pm 0,11 \mathrm{Aab}$ & $1,7 \pm 0,13 \mathrm{Aa}$ & 0,4563 \\
\hline Baicao $^{\circledR}$ & $2,4 \pm 0,50 \mathrm{Aa}$ & $1,8 \pm 0,10 \mathrm{Aa}$ & 0,1767 \\
\hline Topneem & $1,4 \pm 0,10 \mathrm{Bb}$ & $1,9 \pm 0,16 \mathrm{Aa}$ & 0,0164 \\
\hline$P$ & 0,008 & 0,6126 & \\
\hline
\end{tabular}

Médias seguidas pela mesma letra, minúscula na coluna, não diferem entre si pelo teste Kruskal-Wallis $(\mathrm{p}<0,05)$.

Médias seguidas pela mesma letra, maiúscula na linha, não diferem entre si pelo teste Mann-Whitney ( $<<0,05)$.

\section{CONCLUSÕES}

Orobor $^{\circledR}$ é seletivo ao parasitoide $T$. pretiosum, em condições de laboratório.

Baicao $^{\circledR}$ não é seletivo e foi classificado como levemente nocivo a T. pretiosum.

Topneem ${ }^{\circledR}$ causou redução do número de ovos parasitados por $T$. pretiosum, quando o parasitoide tem chance de escolha. Topneem ${ }^{\circledR}$ não interferiu nos demais parâmetros avaliados.

\section{AGRADECIMENTOS}

Os autores agradecem à CAPES, Coordenação de Aperfeiçoamento de Pessoal de Nível Superior, pela concessão da bolsa de pós-graduação à primeira autora.

À professora Dra. Vanda Pietrowski e à M.Sc. Mariana Pizzatto, do Centro de Ciências Agrárias, da Universidade Estadual do Oeste do Paraná - Unioeste, Campus Marechal Cândido Rondon, PR, pelo fornecimento de Trichogramma pretiosum.

\section{REFERÊNCIAS}

Andrade LH, Oliveira JV, Lima IMM, Santana MF \& Breda MO (2013) Efeito repelente de azadiractina e óleos essenciais sobre Aphis gossypii Glover (Hemiptera: Aphididae) em algodoeiro. Revista Ciência Agronômica, 44:628-634.

Ayres M, Ayres Júnior M, Ayres DL, Santos AS \& Ayres LL (2007) BioEstat: Aplicação estatística nas áreas das ciências bio-médicas. Belém, Pará: Sociedade Civil Mamirauá, MCT-CNPq, 324p.

Bestete LR, Pratissoli D, Queiroz VT, Celestino FN \& Machado LC (2011) Toxicidade de óleo de mamona a Helicoverpa zea e a Trichogramma pretiosum. Pesquisa Agropecuária Brasileira, 46:791-797.
Broglio-Micheletti SMF, Santos AJN \& Pereira-Barros JL (2006) Ação de alguns produtos fitossanitários para adultos de Trichogramma galloi Zucchi, 1988 (Hymenoptera: Trichogrammatidae). Ciência Agrotécnica, 30:1051-1055.

Cônsoli FL, Rossi MM \& Parra JRP (1999) Developmental time and characteristics of the immature stages of Trichogramma galloi and T pretiosum (Hymenoptera, Trichogrammatidae). Revista Brasileira de Entomologia, 43:271-275.

Correia AA (2012) Avaliação de inseticidas sobre a biologia e embriologia de Spodoptera frugiperda (J.E. Smith) (Lepidoptera: Noctuidae) e o efeito em Trichogramma pretiosum Riley (Hymenoptera: Trichogrammatidae), parasitoide de ovos. Tese de Doutorado. Universidade Federal Rural de Pernambuco, Recife. 87p.

Correia AA, Wanderley-Teixeira V, Teixeira AAC, Oliveira JV, Golçaves GGA, Cavalcanti MGS, Brayner FA \& Alves LC (2013) Microscopic Analysis of Spodoptera frugiperda (Lepidoptera: NOctuidae) embryonic development before and after treatment eith Azadirachtin, Lufenuron and Deltamethrin. Journal of Economic Entomology, 106:747-755.

Degrande PE, Reis PR, Carvalho GA \& Berlamino LC (2002) Metodologia para avaliar impacto de pesticidas sobre inimigos naturais. In: Parra JRP, Botelho PSM, Corrêa-Ferreira BS \& Bento JM (Eds.) Controle biológico no Brasil: parasitoides e predadores. São Paulo, Manole. p.71-93.

Dequech STB, Egewarth R, Sausen CD, Sturza VS \& Ribeiro LP (2009) Ação de extratos de plantas na oviposição e na mortalidade da traça das crucíferas. Ciência Rural, 39:551-554.

Faria CA, Torres JB, Fernandes AMV \& Farias AMI (2008) Parasitism of Tuta absoluta in tomato plants by Trichogramma pretiosum Riley in response to host density and plant structures. Ciência Rural, 38:15041509 .

Gallo D, Nakano O, Neto SS, Carvalho RPL, Baptista GC, Filho EB, Parra JRP, Zucchi RA, Alves SB, Vendramim JD, Marchini LC, Spotti JR \& Omoto C (2002) Entomologia Agrícola. Piracicaba, FEALQ. 920p.

Gonçalves-Gervásio RCR \& Vendramim JD (2004) Efeito de Extratos de Meliáceas Sobre o Parasitóide de Ovos Trichogramma pretiosum Riley (Hymenoptera: Trichogrammatidae). Neotropical Entomology, 33:607-610. 
Gonçalves JR, Holtz AM, Pratissoli D \& Guedes RNC (2003) Avaliação da qualidade de Trichogramma pretiosum (Hymenoptera: Trichogrammatidae) em ovos de Sitotroga cerealella (Lepidoptera: Gelechiidae). Acta Scientiarum, Agrononomy, 25:485-489.

Hassan AS (1997) Métodos padronizados para testes de seletividade, com ênfase em Trichogramma. In: Parra JRP \& Zucchi RA (ed.) Trichogramma e o Controle Biológico Aplicado. Piracicaba, FEALQ. $324 \mathrm{p}$.

Hohmann CL, Silva FAC \& Novaes TG (2010) Selectivity of Neem to Trichogramma pretiosum Riley and Trichogrammatoidea annulata De Santis (Hymenoptera: Trichogrammatidae). Neotropical Entomology, 39:985-990.

Kathrina GA \& Antonio LOJ (2004) Controle biológico de insectos mediante extractos botânicos. In: Cabrall M \& Guarahay F (Eds.) Controle biológico de plagas agrícolas. Managua, Catie. 232p.

Menezes ELA (2005) Inseticidas botânicos: seus princípios ativos, modo de ação e uso agrícola. Seropédica, Embrapa Agrobiologia. 58p.

MFRural (2011) Topneem Inseticida Natural. Disponível em: <http:// www.mfrural.com.br/detalhe.asp?cdp=51762\&nmoca $=$ top-neem-inseticida-natural>. Acessado em: 08 de março de 2011.

Morandi Filho WJ, Botton M, Grutzmacher AD, Giolo FP \& Manzoni CG (2006) Ação de produtos naturais sobre a sobrevivência de Argyrotaenia sphaleropa (Meyrick) (Lepidoptera: Tortricidae) e seletividade de inseticidas utilizados na produção orgânica de videira sobre Trichogramma pretiosum Riley (Hymenoptera: Trichogrammatidae). Ciência Rural, 36:1072-1078.

Oliveira RC, Pratissoli D \& Bueno AF (2003) Efeito de Azadirachata indica (NIM) sobre o parasitismo de Trichogramma pretiosum (Hymenoptera: Trichogrammatidae) em ovos de Anasgasta kuehniella (Lepidoptera: Pyralidae). Revista Ecossistema, 28:75-78

Parra JRP \& Zucchi RA (2004) Trichogramma in Brazil: Feasibility of use after twenty years of research. Neotropical Entomology, 33:271281.

Penteado SR (2007) Defensivos alternativos e naturais: para uma agricultura saudável. $3^{\mathrm{a}}$ ed. Campinas, Via Orgânica. 172p.

Potrich M, Alves LFA, Haas J, Silva ERL, Daros A, Pietrowski V \& Neves PMOJ (2009) Seletividade de Beauveria bassiana e Metarhizium anisopliae a Trichogramma pretiosum Riley (Hymenoptera: Trichogrammatidae). Neotropical Entomology, 38:822826.
Potrich M (2010) Seletividade de fungos entomopatogênicos a Trichogramma pretiosum Riley (Hymenoptera: Trichogrammatidae) e virulência a Bemisia tabaci (Hemiptera: Aleyrodidae). Tese de Doutorado. Universidade Estadual de Londrina, Londrina. 142p.

Purwatiningsih, Heather N \& Hassan E (2012) Efficacy of Leptospermum petersonii oil, on Plutella xylostella, and its parasitoid, Trichogramma pretiosum. Journal of Economic Entomology, 105:1379-1384.

Sidi MB, Islam T, Ibrahim Y \& Omar D (2012) Effect of insecticide residue and spray volume application of azadirachtin and rotenone on Trichogramma papilionis (Hymenoptera: Trichogrammatidae). International Journal of Agriculture \& Biology, 14:805-810.

Silva ERL (2010) Efeito de produtos alternativos sobre Bacillus thuringiensis subesp. Kurstaki e Trichogramma pretiosum Riley (Hymenoptera: Trichogrammatidae). Tese de Doutorado. Universidade Estadual de Londrina, Londrina. 117p.

Siqueira JR, Bueno RCOF, Bueno AF \& Vieira SS (2012) Preferência hospedeira do parasitoide de ovos Trichogramma pretiosum. Ciencia Rural, 42:1-5.

Smanitto LF, Gouvea A, Potrich M, Silva ERL, Silva J, Pagorini CS (2013). Seletividade de produtos alternativos a Telenomus podisi Ashmead (Hymenoptera: Scelionidae). Semina: Ciências agrárias, 34:3295-3306.

Tavares WS, Cruz I, Petacci F, Assis Junior SL, Freitas SS, Zanuncio JC \& Serrão JE (2009) Potential use of Asteraceae extracts to control Spodoptera frugiperda (Lepidoptera: Noctuidae) and selectivity to their parasitoids Trichogramma pretiosum (Hymenoptera: Trichogrammatidae) and Telenomus remus (Hymenoptera: Scelionidae). Industrial Crops and Products, 30:384-388.

Vinson SB (1997) Comportamento de seleção hospedeira de parasitoides de ovos, com ênfase na família Trichogrammatidae. In: Parra JRP \& Zucchi RA (ed.) Trichogramma e o Controle Biológico Aplicado. Piracicaba, FEALQ. 324p.

Xavier VM (2009) Impacto de inseticidas botânicos sobre Apis melífera, Nannotrigona testaceicornis e Tetragonisca angustula (Hymenoptera: Apidae). Dissertação de mestrado. Universidade Federal de Viçosa, Viçosa. 43p. 